G.H. Watson, R.L. Tucker and J.K. Walters (Editors)

1993 Elsevier Science Publishers B.V.

\title{
Study on Simulation for ROOF PUSHUP CONSTRUCTION METHOD
}

\author{
Masahiro Morita, Assistant Chief Researcher \\ Eiji Muro, Senior Chief Researcher \\ Tetsuo Kanaiwa, Chief Researcher \\ Hiroyuki Nishimura, Researcher
}

Takenaka Technical Research \& Development Institute, 2-5-14 Minamisuna, Koto-ku, Tokyo

\begin{abstract}
In the construction industry, both construction and management technology have been developed, as well as industrization, automation. However, many problems were found in the process of applying such technologies for each construction projects in which it is required to move to the different work place and forced to construct at outside.

Based on this, aiming to improve the environment of the work place, we have developed ROOF PUSHUP METHOD CONSTRUCTION which requires to gather main constructive tasks beneath the top floor, while the construction itself is being managed by the computer, and this method was applied to construction of an office building. This paper describes the outline of ROOF PUSHUP CONSTRUCTION METHOD, and the actual result of applying it. This paper discusses the necessity of the construction simulation system for ROOF PUSHUP CONSTRUCTION METHOD, and the current status and the examples of such simulation systems. Furthermore the paper discusses the outline and the result of applying the simulation system developed based on the actual requirements.
\end{abstract}

\section{Introduction}

To resolve the problem of labor shortage (present and future) in the construction industry, The new construction method (the Roof Push-up Construction Method) has been developed. This method can automatize, robotize, and industrialize various aspects and phases of construction work in all weather conditions. Takenaka Corporation has applied the method to a real construction project and obtained positive results in shorter construction time and manpower savings. To evaluate the benefits of the Roof Push-up Method to determine its applicability, however, a model system for analyzing, simulating and evaluating the method must be devised. Only with such a system will it become possible to determine, in advance, the optimum installation order of construction materials and the most effective material flow.

The purpose of this paper is: (1) to provide general information on the Roof Push-up Method and its application in the Yanagibashi Mitsui Building in Japan and (2) to present a study on simulation in determining the benefits of the Roof Push-up Method.

\section{An Outline of Roof Push-up Construction Method}

\subsection{The Positioning and the Benefit of Roof Push-up Construction Method}

It is obvious that labor shortages will become more and more serious in the future. To overcome this problem, an automated construction method has been devised. In our estimation, a fully automated and industrialized construction method can reduce construction time and the 
number of processes involved $40 \%$ in comparison with the same construction methods and at the same construction cost. With this goal in mind, Takenaka Corporation has developed the Roof Push-up Construction Method (Fig. 2.1), which exemplifics the industrialization of building construction. It unifies various aspects of construction work via robotic, computer science, and automation technologies. With the Roof Push-up Method, a safe and comfortable working environment can be provided under all weather conditions.

The Roof Push-up Method differs from conventional construction methods in methods in which steel frames are assembled up to a specified height, and then the ceiling and floor of each level are assembled. The Roof Push-up Method was applied in construction of the Yanagibashi Mitsui Building in Nagoya in 1990 for the first time.

In this method the roof floor (push-up floor) is constructed first. This is done in the sequence shown in Fig. 2.3. The benefits of benefits of this method are summarized in Fig. 2.4

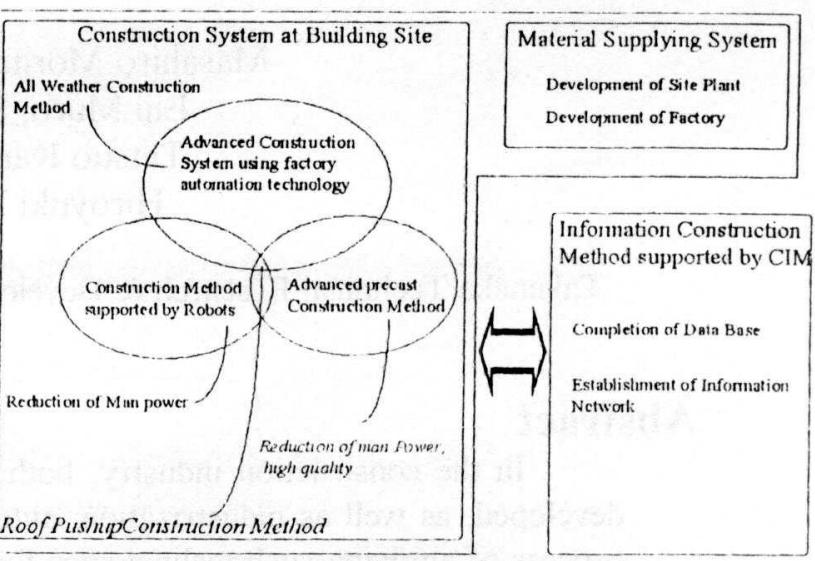

$<$ Fig. 2.1> Conceptual Illustration of the Roof Push-up Method

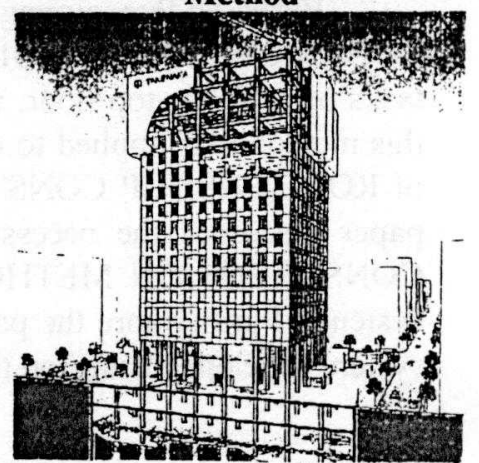

$<$ Fig. 2.2 > Automated Building Construction

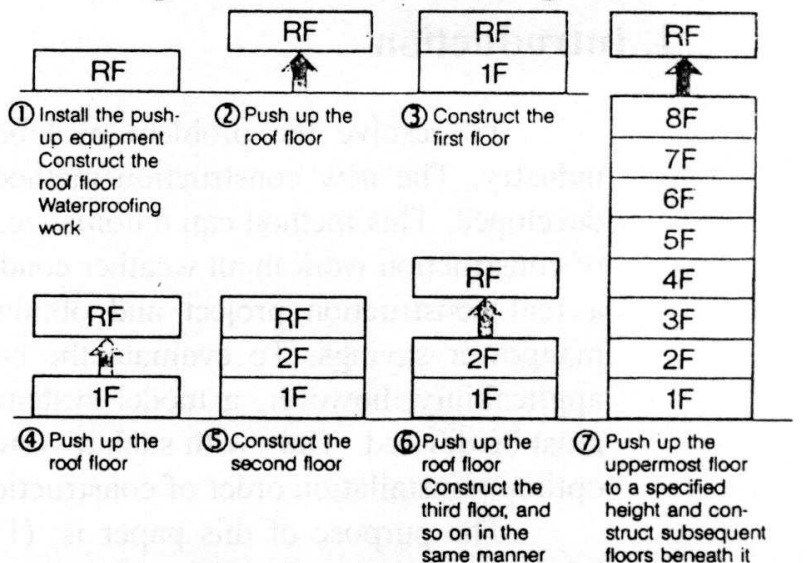

$<$ Fig. 2.3> Sequence and Mechanism of the Roof Push-up Method

(1) Jack-up Mechanism

Our post jacking system has two types of jacks (floor jacks and column jacks).

- The construction work cannot be effected by the weather - Labor is elevated places is reduced. This improves safety - Construction can be automatized due to the integration of various steps of construction.

- The building can be constructed in a narrow or restricted area.

$<$ Fig. 2.4> Benefit of Roof Push-up Method

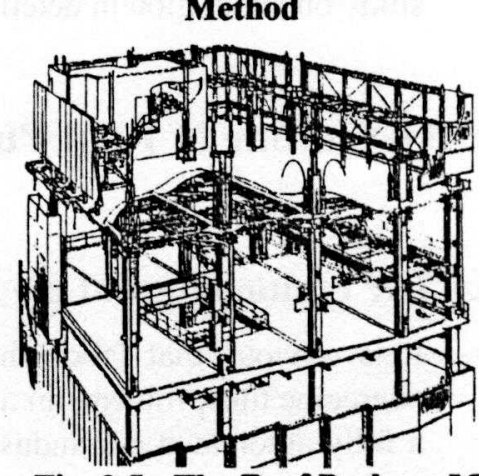

<Fig. 2.5> The Roof Push-up Method 
These jacks are used interchangeably to push up the movable floor (roof floor) as floors below are constructed. As a fixed number of movable columns are jacked up, temporary support columns will be installed. This is to make the building under construction earthquake and wind resistant.

(2) Overhead Circular Gantry Crane System

An overhead circular gantry crane system has been developed to lift and accurately place building frame components for structural support. In addition, to move lifted materials horizontally, Material transporters have also been developed. These transporters are aircushioned and used to deliver construction materials to their destinations.

(3) Central Control System

Operations in the Roof Push-up Method are controlled by a computer system located in the central operation booth on the site. The system consists of three subsystems. The operation monitor subsystem monitors work in progress in the whole construction site. The data from this system is used to inform workers. The communication subsystem is used to give direct instructions to workers working on the movable floor or floors below. The precision monitor subsystem can provide a real-time monitor on floor and column installation.

\subsection{Sequence of the Roof Push-up Method}

First of all, the Roof Push-up Method uses its overhead circular gantry crane system to lift up movable posts, beams, and other materials for the topmost floor. After the materials for the roof portion are assembled, the push-up equipment is installed. Then, facing materials for weatherproofing are installed. The basic installed. The basic construction process after this initial process, are summarized in Fig 2.3.

\subsection{Application Example in Construction of the Yanagibashi Mitsui Building}

(1) Building Outline As shown in Table 2.1, the site is near Nagoya station and surrounded by houses on three sides. Moreover, there was an overhead microwave line above the tower of the building to be constructed. This means

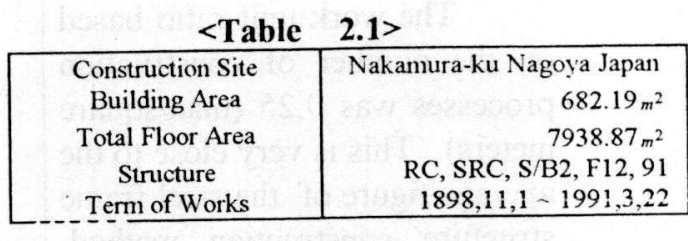

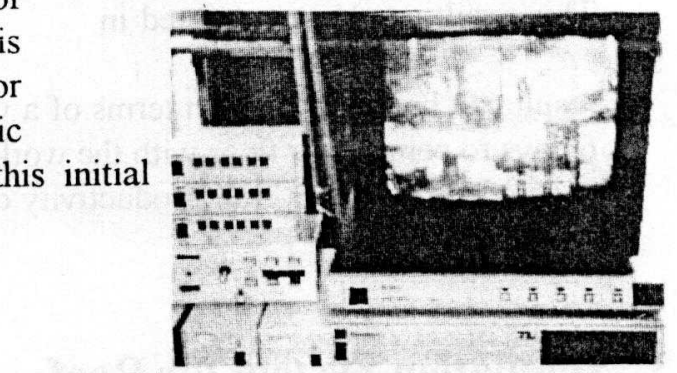

$<$ Photo. 2.1 > Monitoring System

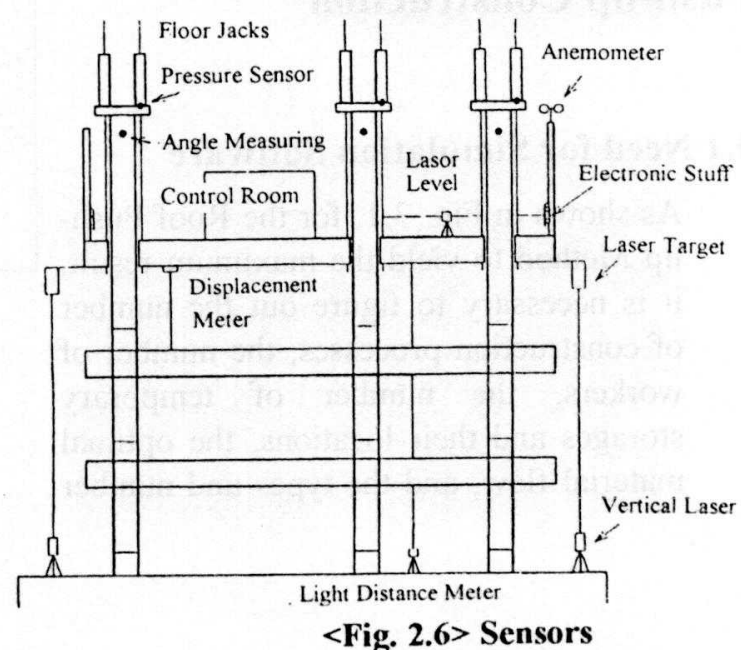

$<$ Fig. 2.6 $>$ Sensors 
that a tower cranecould not be used because of the danger of interfering with the line. As a result, the Roof Push-up Method was adapted to floors 10-12.

By using floor jacks (up-down post jacks), the roof floor (250 tons) was pushed up. Then, wire jacks were used to push up movable posts. After the roof floor was erected, 4 circular gantry cranes were used to install movable columns.

As shown in Photo. 2.1, operations were monitored through remote controlled TV cameras. The precision monitoring was done through sensors installed at various sites in the building (shown in Fig. 2.6). The data was collected in the central operation unit and observed.

(2) Results

The horizontal precision of the movable floor push-up, the vertical precision of the movable column installation, and the floor jack load were all maintained within the set ranges.

The work unit ratio based on the number of construction processes was 0.25 (man/square meters). This is very close to the average figure of the steel frame structure construction method This result can be represented in

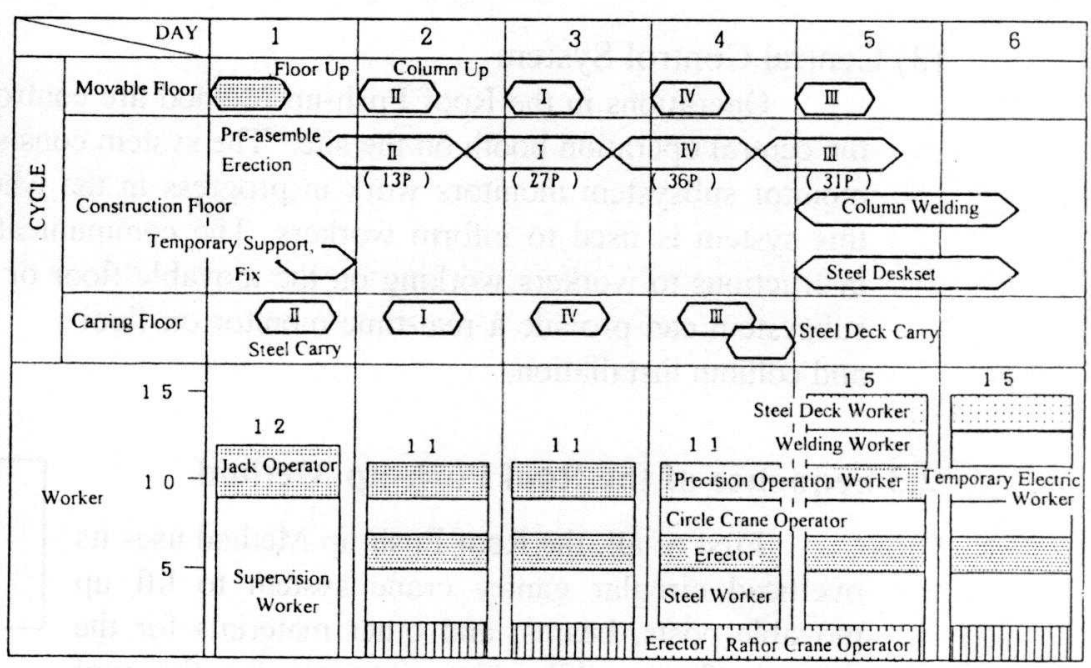

<Fig. 2.7> Overali Work Process Chart

result can be represented in terms of a work process cyclic chart as shown in Fig. 2.7. It takes 6 days to complete a floor with the work unit ratio of 0.17 , in our estimation. Therefore, it was concluded that the overall productivity can be improved.

\section{Simulation System for Roof Push-up Construction}

\subsection{Need for Simulation Software}

As shown in Fig. 3.1, for the Roof Pushup Method to yield the maximum result, it is necessary to figure out the number of construction processes, the number of workers, the number of temporary storages and their locations, the optimal material flow, and the types and number

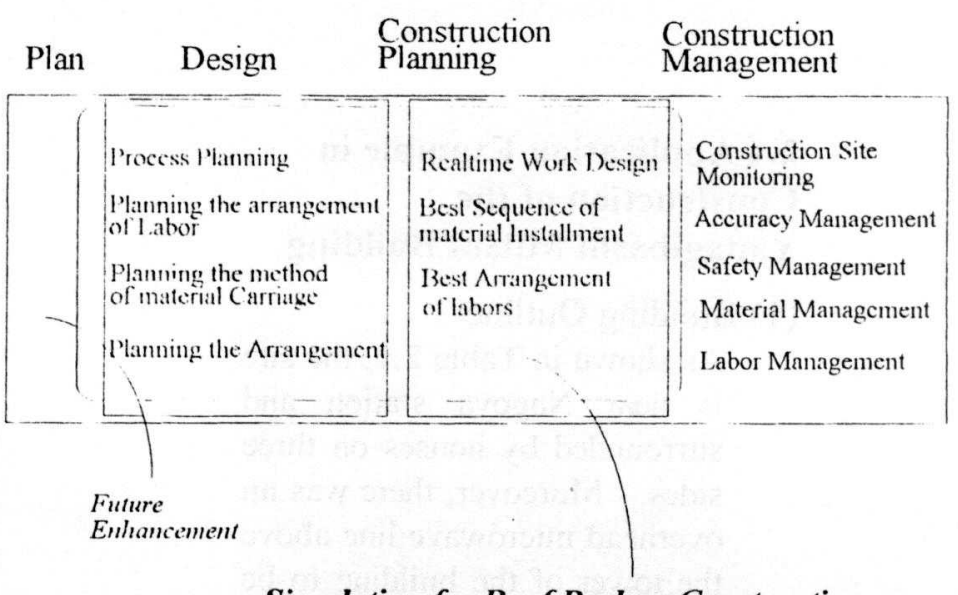

Simulation for Roof Pushup Construction

$<$ Fig. 3.1 $>$ Areal Map for Simulation 
of machines needed for a given construction to obtain the maximum productivity. Since these variables have to be determined prior to the erection of a building, it is necessary to devise a reliable computer software system, by which an application of the Roof Push-up Method under various conditions can be simulated. Such a simulation system, equipped with adequate database, can provide vital information in the future.

\subsection{Special Purpose Simulation System}

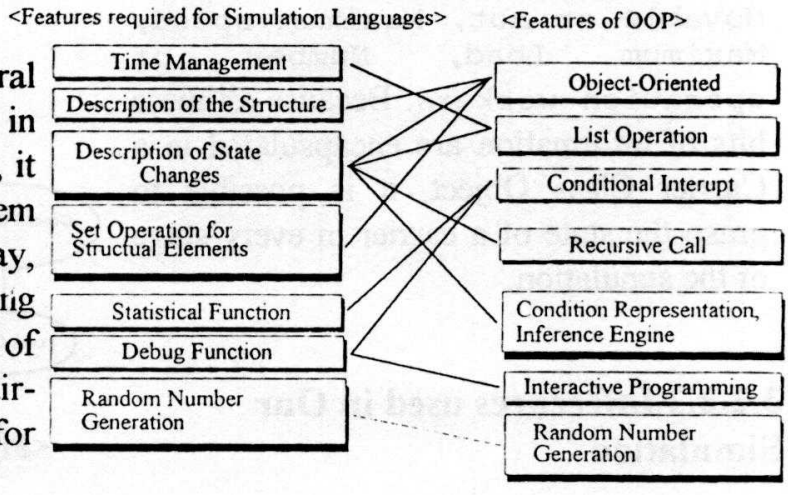

$<$ Fig. 3.2> Functional Correspondence between Simulation Language and Object-Oriented Language

\subsubsection{Language Selection}

Object-Oriented programming (OOP) manipulates objects which can be either base classes or derived classes. Classes are building blocks consisting of variables and functions that associate with variables. Thus, the class mechanism of Object-Oriented programming allows encapsulation of different data types and operations. Derived classes can be created from base classes and other derived classes through inheritance. The foremost benefit of the inheritance mechanism is that it allows for the reuse of existing code to create a new type. In other words, one class is used as a building block for another, which in turn enables a hierarchical representation of knowledge in simulating different models for a given problem. Also the inheritance mechanism can eliminate redundancies in programming. As shown in Fig.3.2, most of the functions needed in our simulation are offered by OOP.

Therefore Common Lisp Object System (CLOS) has been selected for the simulation system. The CLOS was made by reference to Daniel G. Bobbrow's system[2], as none of CLOS package was available.

\subsubsection{Object-Oriented Simulation System}

\subsubsection{Classes for the Simulation}

Considering the ease of maintenance and enhancement, All the simulation entities were defined in terms of classes. For example, every construction material has its unique ID number and its delivery destination (floor number and the attachment position). We need to know whether a material has been delivered to the site or not. The status of delivered material can also vary. Because there can be more than one way for a material to be lifted, it is necessary to keep track of cranes involved in lifting. Because a material has to be joined or connected with other materials, we need to encode how it has to be installed in relation to other materials. All 
these details can be grasped and manipulated easily if simulation entities can be expressed in terms of classes as shown in Fig. 3.3.

In Fig. 3.3, the Carrier class is shown as the top node. This class contains such members as Name of carrier, Movable or not, Maximum speed, Maximum Load, Number of operation workers. Because all these bits of information are encapsulated in a Carrier Class Object, it is possible to grasp the state of a carrier in every stage of the simulation.

\subsubsection{Inferences used in Our Simulation}

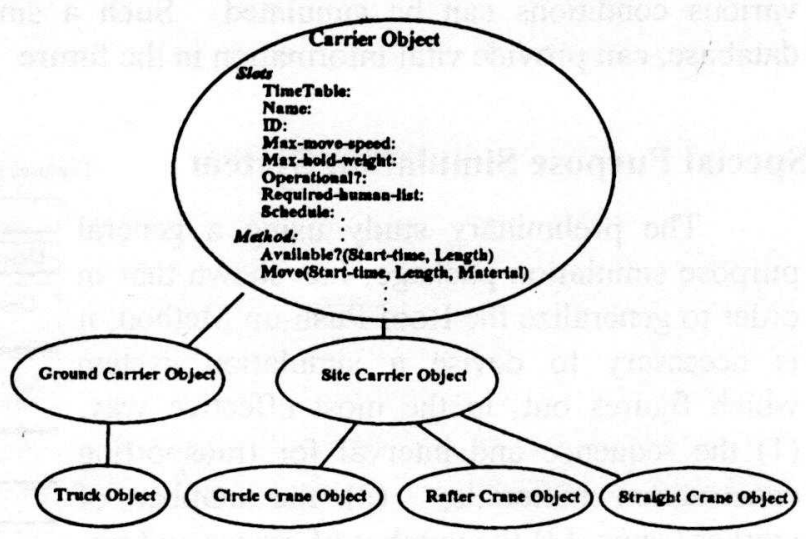

$<$ Fig. 3.3> Example of Object Declarations

Our simulation program performs logical inferences as shown in Fig. 3.4. In other words, upon taking input data specifying the following information, it performs simulation through various inferences to produce an output, which is a work schedule showing the optimum rate of operation for the

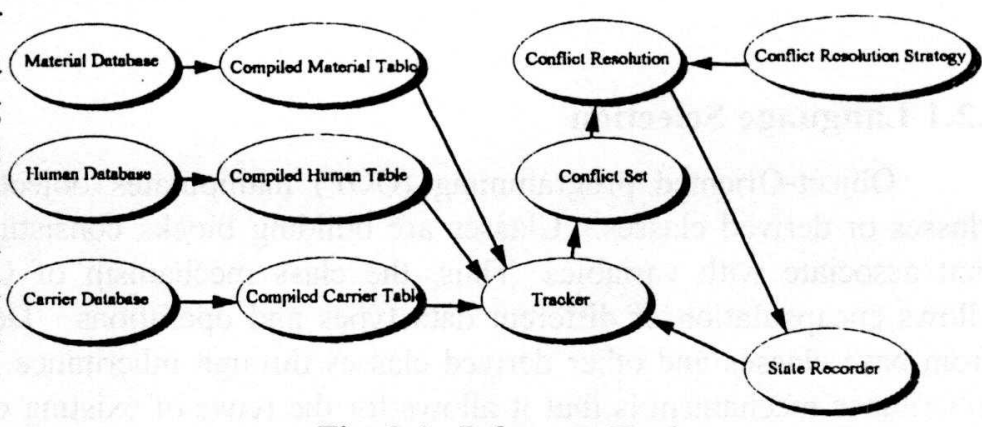

$<$ Fig. 3.4> Inference Engine

cranes, the optimum work unit ratio and the shortest construction period on the given input. For example, the schedule chart in Fig. 3.5 has been obtained by setting the lift-up intervals as 3830 and 1915 seconds and by specifying the number of

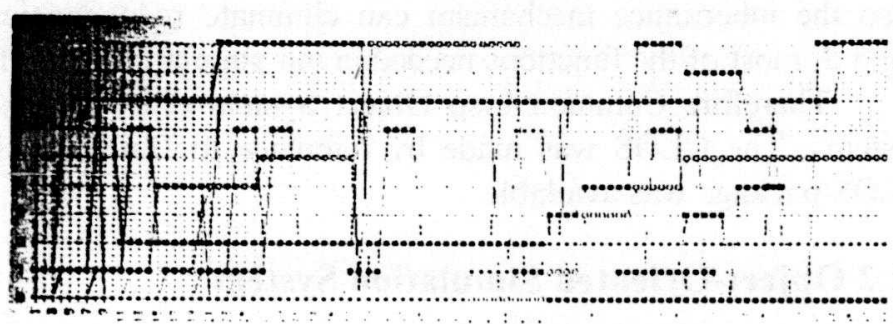

$<$ Fig. 3.5 $>$ Simulated Schedule Chart

worker teams as $1-3$ teams.

Information on construction materials, workers, and machines are compiled into a database which is stored within the simulation program as various types of tables. Our simulation program keeps track of every carrier object. When there is at least one unused crane and there are available workers, liftable materials are grouped for each unused crane to make a combination set of carrier objects. This combination set can be processed in accordance with our inference strategy, i.e., make the selection in such a way that it will shorten the construction period, select the carrier object that is placed furthest from the transporter. Then the specified 
operation of a selected car ier object will be performed and the work progress status record will be updated.

This process will be repeated until all members of the combination set are used. Let us take a more concrete example. Consider the first selection of the day when twenty-nine materials (ID numbers (1) - (29)) are waiting to be lifted up. These twenty-nine items will be selected from the material table to form a combination set. In selecting the first carrier object to be processed, the inference strategy requires consideration of the number of cranes to use, the installation position, the distance from the transporter, the number of workers available, and the installation manner, etc. Suppose ID 1 is selected. Then the portion of the work progress status record for ID 1 will be updated. Now the combination set contains ID numbers (2) - (29). Thus, the whole process will be repeated on that set.

\subsubsection{Simulation Results}

Based on the model used for a commercial simulation package, Simulations under various conditions were performed. It was important to find out (1) the most efficient worker team setup, (2) the number of storable materials which leads to minimization of the daily manpower hours, and (3) the most efficient crane setup to obtain the optimum rate of operation for the cranes. The time needed for the simulation was 18 - 24 minutes on our Macintosh IIfx. Only half of that time was needed on a SPARC2 workstation.

Assuming that it takes 3830 seconds to lift up an installation post, the simulation was performed by altering the number of worker teams, the capacity of the storage place in terms of the number of storable items, and the number and types of cranes available. The simulation results are given in Tables 3.1, 3.2, and 3.3.

\subsubsection{Worker Team Setup}

As shown in Table 3.1 , the amount of completion time with the 10 worker team setup was 5.1 hours. With the 45 worker team setup, it was 3.6 hours. (We postulated 45 is the maximum number of workers we could put in one work team). As far as the manpower per

$<$ Table 3.1 $>$ No. of workers and their time required

\begin{tabular}{|c|cc|}
\hline No of workers & Work time (Hour) & Man-time \\
\hline 1 Team (10) & 5.15 & 51.5 \\
2 Teams (12) & 4.85 & 58.2 \\
3 Teams (15) & 3.99 & 59.9 \\
Unlimited (45) & 3.36 & 128.7 \\
\hline
\end{tabular}
hour is concerned, we concluded that the 10 concerned, we concluded that the 10 worker setup was the most efficient.

\subsubsection{Storage Capacities}

As shown in Table 3.2, with three storable items the amount of time required for completion was in the 10-13 hour-range. When the number of storable items increased to 5 or more, the amount of time required was in the 4-8 hour-range.

$<$ Table 3.2 > Time required depending on No. of workers and Max. No. of items in stock-yields (Hours)

\begin{tabular}{|c|ccc|}
\hline No of workers & $\begin{array}{c}\text { Maximum 3 in } \\
\text { stock-yields }\end{array}$ & $\begin{array}{c}\text { Max } 5 \text { in } \\
\text { stock-yields }\end{array}$ & $\begin{array}{c}\text { Inlimited in } \\
\text { stock-yields }\end{array}$ \\
\hline 1 Team (10) & 13.26 & 8.22 & 7.50 \\
2 Teams (12) & 12.42 & 6.06 & 5.76 \\
3 Teams (15) & 11.34 & 5.52 & 5.16 \\
Unlimited (45) & 10.44 & 3.60 & 3.30 \\
\hline
\end{tabular}

range. Thus, we concluded that the temporary storage place needs to be wide enough to keep at least 5 items at one time. 


\subsubsection{Crane Setups}

$<$ Table 3.3> Time required when different cranes are used by 1 team

\begin{tabular}{|c|c|c|}
\hline $\begin{array}{c}\text { Casel: Use } 2 \text { slide cranes } \\
\text { instead of } 4 \text { circle cranes }\end{array}$ & $\begin{array}{c}\text { Case2: Casel one } \\
\text { Rafter crane }\end{array}$ & $\begin{array}{c}\text { Case 3: } 4 \text { circle crane + } \\
\text { one Rafter crane }\end{array}$ \\
\hline 11.16 & 4.50 & 3.96 \\
\hline
\end{tabular}

As shown in Table 3.3, with the two slide-crane setup, the amount of the crane operation ended up with 11.16 hours. With the two-slide crane and one rafter crane setup, it was 4.5 hours. With the four circular gantry crane and one rafter crane setup, it was 3.96 hours.

\subsubsection{Evaluation of Simulation Results}

The simulation experiment has shown that the productivity of the Roof Push-Up Method depends on the worker team setup and the crane setup. Thus, it was concluded that it is possible to make the Roof Push-Up Method cost-productive by carefully choosing the most effective combination of the worker team setup and the crane setup.

\section{Summary and conclusions}

In this paper Takenaka Corporation's Roof Push-Up Method has been introduced, which offers automation and all-weather operation in building construction. This method was employed partially in the Yanagibashi Mitsui Building construction in Nagoya, Japan. From that trial, it was confirmed that the Roof Push-Up Method can result in shorter construction time and manpower savings. The Yanagibashi Mitsui project also showed that the method can be augmented with a simulation system capable of figuring out, in advance, the optimum material installation sequence and the optimum material delivery routes. Therefore, a simulation system was developed in CLOS to take care of the problems which became clear in the preliminary simulation study with a commercial simulation package. Our CLOS simulation experiment confirmed that the Roof Push-Up Method can be made effective and productive by choosing parameters that affect the crane setup and the worker team setup.

The developed simulation program offers a reasonable foundation for further design improvement. Currently, the following areas of enhancement are being prepared in order to make a program capable of a real-time simulation (limiting the maximum time required at 3 minutes).

- Development of tables for data indicating installation start time;

- Development of a parallel processing system for inferences;

Also planned is a graphic user interface which can make the simulation system more user-friendly and practical.

$<$ References $>$

1) ISTEL: "WITNESS User Manual Version 6.0 Issue 2", 1989

2) Daniel G. Bobrow: "Common Lisp Object System Specification", ANSI at the X3J13, 1988

3) APLLE Computer, INC.: "Allegro Common Lisp ${ }_{\mathrm{TM}} 1.4$ ", 1990 